\title{
El avance de la deforestación en México 1976-2007
}

\section{The progress of the deforestation in Mexico 1976-2007}

\author{
Fernando A. Rosete-Vergés', José Luis Pérez-Damián', Mariano Villalobos-Delgado², Elda N. Navarro-Salas³, \\ Eduardo Salinas-Chávez y Ricardo Remond-Noa ${ }^{4}$
}

1 Instituto Nacional de Ecología y Cambio Climático, México. farosete@yahoo.com.mx, jldamian@ine. gob.mx

\author{
2 Instituto Nacional de Estadística y Geografía, \\ México. \\ 3 Instituto de Geografía. UNAM. México. \\ 4 Facultad de Geografía. Universidad de La Habana. \\ cuba. esalinas@geo.uh.cu
}

\section{RESUMEN}

El presente trabajo busca conocer el avance de la deforestación en México, a partir del análisis del cambio de uso de suelo y vegetación entre la Serie I, 1976, y la Serie IV, 2007, del INEGI. Se incorporan los resultados obtenidos en el Inventario Nacional Forestal del 2000 para hacer un corte por sub-periodos (1976-2000 y 2000-2007). Además, se realiza un análisis al nivel de polígono sobre la dinámica de las superficies forestales con vegetación secundaria, con la finalidad de establecer si se trata de un proceso de recuperación o de alteración forestal. Los resultados encontrados demuestran que las tasas de cambio han disminuido en el periodo 2000-2007, pero que la superficie forestal disminuye anualmente en alrededor de 500000 ha. Para el periodo 1976-2007, se encontró una pérdida anual de 534707 ha. Los datos oficiales de deforestación muestran una subvaluación a partir de 2006, ya que se descuenta 100\% de la superficie ocupada por vegetación secundaria, cuando en la realidad el proceso de recuperación es de entre $10 \%$ y $13 \%$ de dicha superficie. Con el dato ajustado del proceso de recuperación se recalcularon las cifras oficiales publicadas a partir de 2006, mostrando consistencia en relación con la estabilización de la superficie anual deforestada. El destino principal de la superficie deforestada sigue siendo la agricultura de temporal.

PALABRAS CLAVE: Alteración forestal, cambio de uso del suelo, proceso de recuperación, tasas de cambio, vegetación secundaria.

\section{ABSTRACT}

This paper seeks to show the deforestation rate in Mexico based on land use and vegetation changes data from 1976 to 2007 using the official data published by INEGI. The results of the National Forest Inventory for 2000 were added to make a cut and the process was analyzed in two stages from 1976 to 2000 and from 2000 to 2007. Moreover, a polygon level analysis was carried out over the forest with secondary vegetation to know if they are coming from a recovery process or from a degradation process. The results show that the change rates are lower in the 2000-2007 than the 1976-2000 periods, but the annual loss of forest are around the 500000 ha. For the complete period it was found a forest annual loss of 534707 ha. The deforestation official data are sub valued since 2006 because they discount the $100 \%$ of secondary vegetation, when the real amount of recovery process is over $10 \%$ to $13 \%$. The deforestation official data were recalculated from 2006 on, and the new results match with the amount of 500000 ha per year. The principal use for the deforested areas is still the rainfed agriculture.

KEY WORDS: Forest alteration, land use change, recovery process, change rates, secondary vegetation. 


\section{INTRODUCCIÓN}

Los procesos negativos de cambio de uso del suelo y vegetación han sido documentados como el segundo problema ambiental a nivel global (Xiao et al., 2006), ya que afectan el capital natural (Srivastava, 2002; Brooks et al., 2006), la estabilidad microclimática, la dotación de servicios ambientales (Dunjó et al., 2003; Jianchu et al., 2005; Milesi et al., 2005; Musaoglu et al., 2005; Chikhaoui et al., 2005; Heistermann et al., 2006; Lambin y Geist, 2006; Rudel et al., 2005) y el incremento de la concentración de los gases de efecto de invernadero en la atmósfera (Kerr et al., 2003; Campos et al., 2004; Xiao et al., 2006; Clark 2009), además de limitar a nivel local las posibilidades de realizar un manejo sustentable del territorio y sus recursos naturales (Ehrlich y Ehrlich, 2013).

Los estudios más recientes se han centrado en las repercusiones de esos procesos en el cambio climático global y los resultados expuestos sugieren, aunque a veces en forma contrapuesta, que en el caso de México, si se reducen las tasas de deforestación y degradación forestal a la mitad de las reportadas por FAO (2010) (0,5 y 1,2 respectivamente), en los próximos 10 años se podría evitar la emisión de 117 millones de toneladas de carbono a la atmosfera por no cambiar el uso del suelo en las áreas forestales (CABAL, 2010). Incluso ahora se le da más importancia por su contribución al incremento de la sequía y la alteración de las condiciones hidroclimáticas en el territorio (Dai, 2011).

En México, hay pocos estudios a nivel nacional para conocer los procesos de cambio de uso de suelo y vegetación (Castillo et al., 1989; Masera et al., 1992; Mas et al., 1996; Velázquez et al., 2002; Mas et al., 2004; Sánchez et al., 2009; Velázquez et al., 2010), así como algunos otros de enfoque continental con referencia a nivel de país (FAO, 1988; Repetto, 1988; Myers, 1989; WRI, 1992 y 1994), pero a nivel regional y local existen muchos de ellos que han utilizado diferentes enfoques y objetivos, siendo una característica casi generalizada la carencia del análisis de las causas que provocan el cambio (Rosete, 2008 y Velázquez, 2008).

A partir de la elaboración del Inventario Nacional Forestal y de Suelos (INFyS) 2004-2009 (Conafor, 2012a), la Conafor y la Semarnat se encargaron de analizar los procesos de cambio de uso de suelo y vegetación a nivel nacional, publicando que la tasa de deforestación anual ha disminuido drásticamente en los últimos años, en comparación con las tasas calculadas para periodos anteriores a 2004 (p. ej. Masera et al., 1992; FAO, 1995; Velázquez et al., 2002), año de adquisición de los datos nacionales con los que se elaboró el INFyS 2009 (Conafor, 2012a).

Sin embargo, al analizar los resultados más recientes (Conafor, 2012b y Sánchez et al., 2009), es evidente que los procesos de deforestación continúan, pero la diferencia la hace la recuperación de la vegetación, sea por procesos naturales (regeneración natural forestal y sucesión secundaria) o artificiales (plantaciones forestales con propósitos comerciales o de restauración). Esos procesos de recuperación de la vegetación, documentados en los análisis previos de cambio de uso del suelo y vegetación a nivel nacional, eran bastante limitados, por lo que, al realizar un análisis más minucioso de los datos presentados por Conafor (2012b) y Semarnat (2006), se encontró que se contabilizaba como recuperación toda la superficie etiquetada como vegetación secundaria.

Algunos estudios han mostrado que las áreas con vegetación secundaria en México, más que derivar de zonas sin vegetación aparente, agrícolas o pecuarias, son el resultado de la alteración de la vegetación primaria o de bosques secundarios, por lo que están más relacionadas con procesos de degradación de la vegetación forestal que de recuperación de la misma (Rosete et al., 1997; Sánchez et al., 2003 y Cuevas et al., 2010).

A finales de la década de 1980 surgió una gran preocupación para conocer el ritmo de la deforestación en México, ya que varios estudios locales y regionales (SARH, 1984; Cortez-Ortiz, 1990; Dirzo y García, 1991; Cuarón, 1991, entre otros), arrojaron tasas de deforestación muy altas, incluso superiores a 10\% anual (SARH, 1984), situación respaldada por el informe de la FAO sobre el estado de los recursos forestales en los países en desarrollo (FAO, 1988). Esos resultados encendieron los focos rojos entre los investigadores preocupados por el tema, por lo que se realizaron diferentes análisis de la pérdida de cobertura forestal a 
nivel país. Entre esos análisis destacan los elaborados por Toledo (1989), Castillo et al. (1989) y Masera et al. (1992). $\mathrm{Y}$ aunque los resultados generados en cada estudio ofrecen tasas de pérdida de la vegetación forestal diferentes, existe cierta consistencia, entre algunos de ellos, en calcular la pérdida anual de vegetación forestal en México alrededor de 700000 hectáreas.

Para tratar de aclarar la incertidumbre generada por el amplio rango de los resultados obtenidos, entre otros objetivos, se planteó la necesidad de hacer un Inventario Nacional Forestal (INF), mismo que se elaboró entre 1992 y 1994 (Conafor, 2012a) y que arrojó nueva información para calcular la deforestación a nivel nacional. Con la información de ese INF, la FAO publicó en 1995 un incremento de la superficie deforestada al año en México, ubicándola en 678000 ha (FAO, 1995). Sin embargo, en el informe de la FAO de 1997 (FAO, 1997) se comienza a identificar una tendencia de disminución en la velocidad de la deforestación al calcular la pérdida anual de bosques en 508000 hectáreas.

Para actualizar el Inventario Nacional Forestal (INF) de 1994, y aclarar la tendencia publicada por la FAO, en el año 2000 se iniciaron los trabajos del nuevo inventario forestal. Con los resultados obtenidos (Palacio et al., 2000) se realizó una comparación de los cambios entre la Serie I del INEGI y el INF 2000, así como entre la Serie II de INEGI (elaborado con información de 1993) y el INF 2000 (Velázquez et al., 2002; Mas et al., 2004), encontrando que la velocidad del cambio identificado es mayor en el periodo 1993-2000 que en el de 1976-2000. Los nuevos resultados arrojados por el INF 2000 no eran halagadores, por lo que rápidamente la Conafor tomó cartas en el asunto y en el año 2004 comenzaron con el levantamiento de la información para el nuevo inventario, que finalmente fue publicado en el año 2012 (Conafor, 2012a). Los datos del INF 2000 fueron cuestionados en el sentido de que no era un verdadero inventario de los recursos forestales, ya que solamente se ejecutó la primera parte del proyecto planteado, la elaboración de una carta de usos del suelo y vegetación a nivel nacional. Sin embargo, la calidad de los datos del INF 2000 fue evaluada por Mas et al. (2009), encontrando que la información generada es confiable.
De esa forma, la Conafor, de acuerdo a lo dispuesto en la Ley General de Desarrollo Forestal Sustentable, emitida en el año 2003, asume la responsabilidad de realizar los INF y de actualizarlos cada 5 años. Hasta el momento, solamente se ha elaborado el INFyS 2009 durante el periodo 2004-2009, mismo que fue publicado en el año 2012 (Conafor, 2012a). En el año 2005 el INEGI publicó su Serie III (datos del 2002) de uso de suelo y vegetación (INEGI, 2005). Los resultados presentados por el INEGI se vieron plasmados en el informe de la FAO de 2005 (FAO, 2005) y en el Programa Sectorial de Medio Ambiente y Recursos Naturales del sexenio 2007-2012 (Semarnat, 2007), en donde se establece una pérdida anual de 260000 ha de bosques y selvas para el periodo 2000-2005 (348 000 ha para el periodo 1990-2000). A partir de la publicación de la Carta de uso del suelo y vegetación Serie IV por parte del INEGI (INEGI, 2010), se calculó el dato más reciente de la pérdida de cobertura forestal en bosques y selvas de México, que es publicado por FAO (FAO, 2010) y por la Presidencia de la República (Presidencia, 2012 y 2011): 155000 ha anuales, en promedio.

$\mathrm{Al}$ analizar los datos más recientes presentados por Semarnat (2007) y Conafor (2012b), el contraste con los datos publicados, como resultado de estudios académicos, es mayúsculo. Por ejemplo, si se toma el dato presentado en el Segundo estudio de País (Sánchez et al., 2009), 486000 ha, que fue obtenido con las mismas fuentes oficiales, se encuentra más de dos veces por encima que la cifra oficial con la que cerró el sexenio 2006-2012, 155000 ha, aunque esa cifra se refiere exclusivamente a bosques y selvas.

Para tratar de entender en dónde estriba la diferencia entre las cifras oficiales y las mostradas por estudios académicos, se hizo una revisión a detalle de las estadísticas presentadas desde la existencia de la Serie III de INEGI (INEGI, 2005) por las dependencias oficiales, encontrándose que, desde la publicación de La gestión ambiental en México (Semarnat, 2006), se inició con la práctica de descontar, es decir, restar, las hectáreas correspondientes a vegetación secundaria del total de las hectáreas deforestadas (Semarnat, 2006. Tabla 7.7, p. 119), de tal modo que en lugar de hablar de 523639 ha deforestadas al año, se da el total de 
337287 ha, ya que se le restan las 186351 ha correspondientes a vegetación secundaria. Resalta cómo cifras oficiales calculadas con los mismos datos arrojan resultados diferentes: 260000 ha (FAO, 2005 y Semarnat, 2007), 337287 ha (Semarnat, 2006), y 235000 ha (Conafor, 2012b). Parte de esas diferencias se puede explicar por los conceptos y métodos utilizados por Conafor, ya que en sus cifras solamente incluye bosques y selvas, y contabiliza como bosques o selvas las áreas con un mínimo de $10 \%$ de cobertura de copa (Conafor, 2012a).

De esa forma, se identificó que existe un problema conceptual básico para poder decidir si se suma, se resta o se incluye en otra categoría, como alteración forestal, la superficie que presenta vegetación secundaria arbórea y arbustiva. Desde un punto de vista teórico, la revegetación de una superficie con vegetación secundaria es un proceso natural de sucesión vegetal a partir de una apertura en el dosel forestal o de la remoción de la vegetación original (Spurr y Barnes, 1982). Sin embargo, en la práctica, lo que sucede es que las superficies ocupadas con vegetación secundaria, más que responder a un proceso de recuperación vegetal, corresponden a un proceso de alteración de las comunidades forestales (Masera et al., 1992; Masera, 1996; Mas et al., 2002; García-Barrios et al., 2009 -para casos nacionales-, y Dirzo y García, 1991; Álvarez-Icaza et al., 1993; Rosete et al., 1997; Cortina et al., 1999; Trejo y Dirzo, 2000; Bocco et al., 2001; López et al., 2001; Sánchez et al., 2003, Velázquez et al., 2003; Rosete et al., 2008; Cuevas et al., 2010; Garibay y Bocco, 2012, entre otros -para casos regionales).

\section{OBJETIVOS}

Los objetivos del presente trabajo fueron: 1. Calcular las tasas de cambio de uso del suelo y vegetación para el periodo de 1976 (Serie I de INEGI) a 2007 (Serie IV de INEGI). 2. Utilizar al INF 2000 (Mas et al., 2002) como fecha intermedia, con la finalidad de conocer si existe diferencia en las tasas si se calculan en dos periodos (19762000 y 2000-2007). 3. Confirmar que las tasas de cambio han disminuido. 4. Identificar si la superficie etiquetada como vegetación secundaria en el 2007 responde a un pro- ceso de recuperación de la cobertura vegetal, y 5. Comparar los resultados obtenidos con las cifras oficiales existentes.

\section{MATERIALES Y MÉTODOS}

La metodología se desarrolló bajo el enfoque geográfico espacial de los mapas digitales de vegetación y uso del suelo de los años 1976 y 2007, escala 1:250 000, generados por el INEGI, existentes en el acervo de información de la Dirección General de Investigación de Ordenamiento Ecológico y Conservación de los Ecosistemas del Instituto Nacional de Ecología, conocidas en el mercado cartográfico como Serie I (INEGI, 2000) y Serie IV (INEGI, 2010), respectivamente, apoyado en las funciones analíticas de superposición, así como de las aplicaciones de generalización espacial del software SIG ArcInfo, versión 9.3.

A continuación se describe el proceso metodológico fundamental que se utilizó para obtener la cartografía digital de la dinámica de la vegetación y del uso del suelo, de 1976 a 2007, útil para reconocer la manifestación territorial de los patrones de cambio de la cobertura vegetal y de los usos del suelo.

\section{Selección de atributos}

Para este análisis espacial se eligieron de cada una de las series los atributos: tipo y desarrollo de la vegetación (con predominio de vegetación primaria o secundaria) por considerar que éstos en conjunto proporcionan el máximo nivel de detalle a la escala 1:250 000 .

\section{Agrupación de información}

Debido a que la Serie I (original) presenta 52 tipos de vegetación y 9 usos del suelo, además de áreas sin vegetación aparente y cuerpos de agua; y que la Serie IV (original) presenta 56 tipos de vegetación y 10 usos del suelo, además de áreas sin vegetación aparente y desprovisto de vegetación y cuerpos de agua, se procedió a agrupar la información de acuerdo con la propuesta de clasificación jerárquica por tipo de vegetación de Velázquez et al. (2002), en 19 grupos (Tabla 1). Once grupos de vegetación están divididos en: con predominio de vegetación primaria y con predominio de vegetación secundaria. 
TABla 1. Clasificación de la vegetación utilizada. Modificada a partir de Velázquez et al. (2002).

Formación

Tipo de vegetación y uso del suelo

Agricultura de riego y humedad

Cultivos

Agricultura de temporal

Plantación forestal

Coníferas

Bosques

Caducifolia y subcaducifolia

Mezquital

Selvas

Perennifolia y subperennifolia

Mezquital

Matorral
Matorral xerófilo

Comunidad y otras coberturas

Agricultura de riego (incluye riego eventual)

Agricultura de humedad

Riego suspendido

Pastizal cultivado

Agricultura de temporal'

Plantación forestal

Bosque de táscate 2,3

Bosque de oyamel (incluye ayarín y cedro) 2,3

Bosque de pino ${ }^{2,3}$

Matorral de coníferas ${ }^{2,3}$

Bosque bajo-abierto 2,3

Bosque pino-encino (incluye encino-pino) ${ }^{2}$

Bosque de encino ${ }^{2,3}$

Bosque mesófilo de montaña 2,3

Selva alta y mediana perennifolia ${ }^{2,3}$

Selva baja perennifolia 2,3

Selva alta y mediana subperennifolia ${ }^{2,3}$

Selva baja subperennifolia 2,3

Selva mediana caducifolia y subcaducifolia ${ }^{2,3}$

Selva baja caducifolia y subcaducifolia ${ }^{2,3}$

Matorral subtropical ${ }^{2,3}$

Selva baja espinosa ${ }^{2,3}$

Mezquital (incluye huizachal) 2,3

Matorral crasicaule 4,5

Matorral sarcocrasicaule ${ }^{4,5}$

Matorral sarcocaule 4,5

Matorral sarcocrasicaule de neblina 4,5

Matorral desértico micrófilo 4,5

Matorral desértico rosetófilo 4,5

Matorral rosetófilo costero 4,5

Vegetación de desiertos arenosos

Matorral espinoso tamaulipeco ${ }^{4,5}$

Matorral submontano ${ }^{4,5}$

Chaparral 4,5

Pradera de alta montaña

Pastizal

Pastizal

Pastizal inducido

Vegetación hidrófila

Vegetación hidrófila

Otros tipos de vegetación

Otros tipos de vegetación

Área sin vegetación aparente

Asentamiento humano

Otras coberturas

Acuacultura

Cuerpo de agua

\section{Pastizal natural (incluye pastizal-huizachal)}

Sabana

Pastizal inducido

Manglar

Popal-tular

Vegetación de galería (incluye bosque y selva)

Palmar

Vegetación halófila y gipsófila

Vegetación de dunas costeras

Área sin vegetación aparente

Asentamiento humano

Acuacultura

Cuerpo de agua

1 Incluye dos categorías: con cultivos anuales o con cultivos permanentes y semipermanentes.

2 Incluye la vegetación primaria y la vegetación secundaria arbórea en una sola categoría.

3 Incluye a las comunidades con vegetación secundaria arbustiva y herbácea.

4 Se refiere a las comunidades de matorrales con vegetación primaria.

5 Incluye la vegetación secundaria derivada de la alteración de los matorrales. 


\section{Superposición espacial o cruce de mapas}

Este procedimiento consistió en la manipulación geométrica-temática de las entidades geográficas de las dos series de vegetación, apoyada en las herramientas de análisis espacial del software SIG ArcInfo versión 9.3, con el fin de que el mapa resultante reflejara la fusión de la geometría de ambos productos, así como el par de atributos útiles para la identificación de los cambios de la vegetación y del uso del suelo del periodo bajo estudio.

\section{Generalización cartográfica por área mínima cartografiable}

Bajo el argumento que "las designaciones cartográficas no reflejan las particularidades y los detalles de poca importancia, propios de uno y otro objeto, sino que destacan los caracteres fundamentales" (Salitchev, 1981), este paso se llevó a cabo para resolver la atomización del mapa, gene- rada principalmente por el efecto de borde cartográfico, seleccionando en él aquellas entidades geográficas de importancia secundaria o escasa (con superficie inferior o igual a $0,25 \mathrm{~km}^{2}$ ) y asignándolas (a manera de fusión) a las entidades con las que éstas compartían mayor superficie. De esta forma se mejoró sustancialmente la calidad de la cartografía, tanto para su visualización como para su análisis estadístico.

\section{Análisis de resultados}

A partir de los atributos: área, tipo y desarrollo de vegetación de 1976 y 2007, se generó una tabla de frecuencias o de combinaciones para conocer la superficie ocupada (en $\mathrm{km}^{2}$ ) para cada cambio de vegetación y uso del suelo que se presentaron durante el periodo de análisis. Con dicha información se armó una matriz de transición para reconocer los patrones del cambio de la vegetación y el uso del suelo.

TABLA 2. Conceptos utilizados en el presente trabajo.

\begin{tabular}{|c|c|}
\hline Concepto & Definición \\
\hline Alteración & Proceso mediante el cual se afecta la estructura y composición de un ecosistema \\
\hline Cambio & $\begin{array}{l}\text { Cuando ocurren cambios en la vegetación y uso del suelo que representan una modificación importante de } \\
\text { la vegetación o el uso del suelo y pueden ser considerados como irreversibles a corto y mediano plazo }\end{array}$ \\
\hline Deforestación & Proceso de pérdida o remoción de la vegetación forestal \\
\hline Degradación & $\begin{array}{l}\text { Proceso mediante el cual se disminuyen las capacidades, funciones, composición o estructura de un ecosis- } \\
\text { tema }\end{array}$ \\
\hline Pérdida & $\begin{array}{l}\text { Cuando se pierde la vegetación primaria o secundaria por diversas causas y este suceso se considera como } \\
\text { muy difícil de revertir a mediano y largo plazo }\end{array}$ \\
\hline Permanencia & $\begin{array}{l}\text { Cuando se mantiene el mismo tipo de vegetación y uso del suelo a través del tiempo en un lugar determi- } \\
\text { nado }\end{array}$ \\
\hline Recuperación & $\begin{array}{l}\text { Cuando se pasa de un tipo inferior de vegetación o uso del suelo a uno superior; o cuando se pasa de agri- } \\
\text { cultura, pastizal o áreas sin vegetación a algún tipo de vegetación secundaria }\end{array}$ \\
\hline Reforestación & Acción de recuperar la cobertura arbórea en un sitio determinado \\
\hline Revegetación & Acción de recuperar parte o toda la cobertura vegetal en un sitio específico \\
\hline Sucesión secundaria & $\begin{array}{l}\text { El remplazo de la biota en un área determinada subsecuente a una perturbación que tiende a interrumpir } \\
\text { más que a destruir una comunidad biótica existente }\end{array}$ \\
\hline Vegetación forestal & Vegetación en la que predominan las plantas leñosas con más de 3 metros de altura \\
\hline Vegetación secundaria & $\begin{array}{l}\text { Calificativo de la vegetación influida directa o indirectamente por alteraciones en la vegetación original o } \\
\text { primaria de un ecosistema. Generalmente está asociada al proceso de sucesión secundaria }\end{array}$ \\
\hline
\end{tabular}




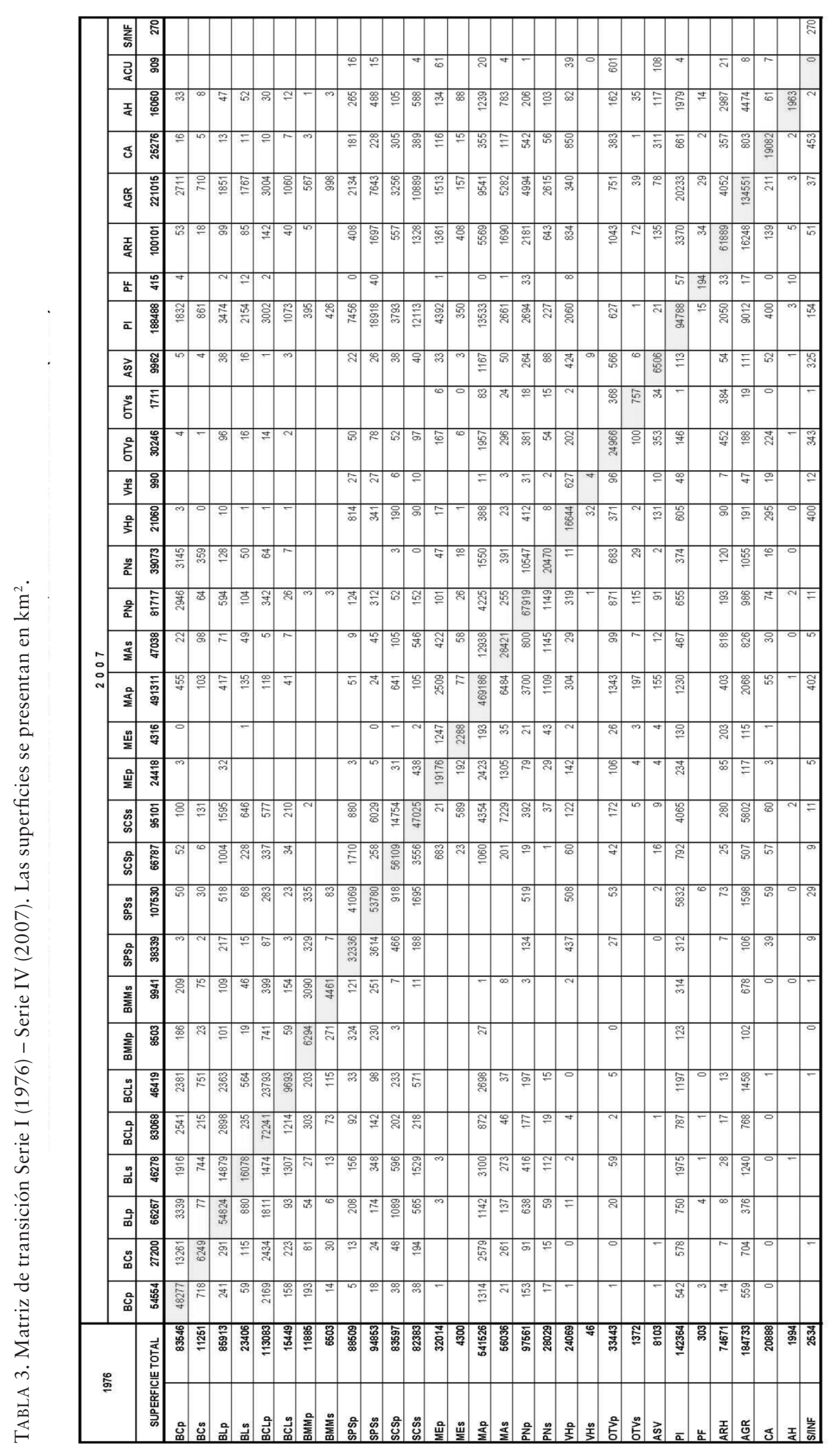

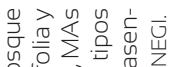

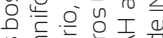

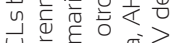

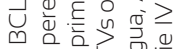

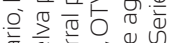

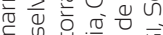

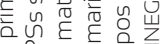

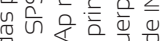

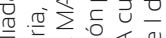

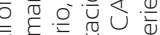

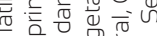

两

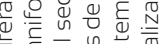

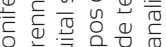

v

ब जै है

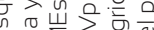

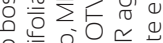

Q

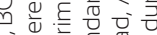

은

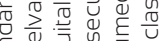

un

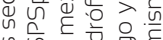

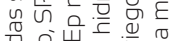

西遂 $\sum$ :

은 줃. 준.

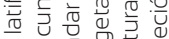

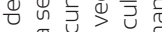

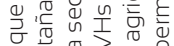

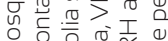

ह

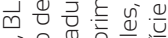

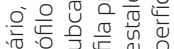

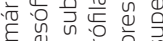

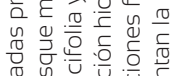

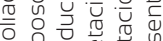

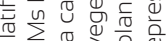

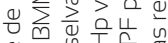

等

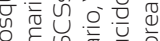

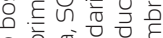

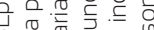

“

을 는

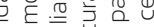

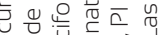

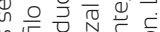

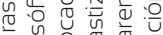

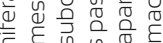

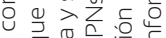

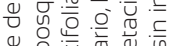

d

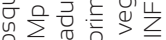

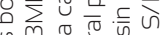

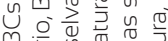

o 西

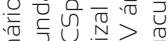

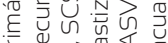

各 un

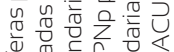

흔

要

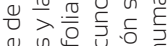

势

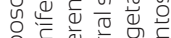

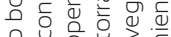

Qs: 
Con la matriz de transición se calcularon las tasas de cambio a partir de la fórmula de FAO (FAO, 1996).

$$
t=(S 2 / S 1)^{1 / n}-1
$$

Donde:

$t=$ Tasa de cambio

$S 1$ = Superficie en la fecha 1

$S 2$ = Superficie en la fecha 2

$n=$ Número de años entre las dos fechas

Para conocer si efectivamente la superficie identificada con vegetación secundaria en la Serie IV (INEGI, 2010) es resultado de un proceso de recuperación vegetal, se realizó un análisis al nivel de polígono con esa condición de la vegetación para conocer de dónde provienen esas superficies. Para tener una mayor consistencia en el análisis, también se identificó a qué categoría pasaron, en la Serie IV, los polígonos etiquetados con vegetación secundaria en la Serie I (INEGI, 2000). En la tabla 2 se presentan los conceptos utilizados en el presente trabajo.

\section{RESULTADOS}

En la tabla 3 se presenta la matriz de transición para el periodo analizado (1976 y 2007). Resalta la disminución en la superficie cubierta por bosques y selvas durante el periodo, así como el notable incremento de la superficie cubierta por agricultura, pastizales y asentamientos humanos.

Otro resultado notorio es que en la superficie con cobertura forestal la vegetación primaria ha disminuido, mientras que los diferentes tipos de vegetación que presentan vegetación secundaria se han incrementado. En general, en números absolutos, de 1976 a 2007 se deforestaron 16575924 hectáreas, lo que en promedio es una pérdida de vegetación en alrededor de 534707 ha al año.

Durante ese mismo periodo de tiempo, 15695140 ha de vegetación primaria pasaron a tener una condición de vegetación secundaria, lo que en promedio representa una alteración de 506295 ha al año de vegetación primaria. Vale la pena resaltar que la superficie cubierta con vegetación secundaria pasó de $323627 \mathrm{~km}^{2}$ en 1976 a 425596 $\mathrm{km}^{2}$ en 2007 , lo que representa un incremento de casi la

TABLA 4. Tasa anual de cambio 1976-2007 por formación vegetal. Las superficies se presentan en $\mathrm{km}^{2}$.

\begin{tabular}{lcccc}
\hline & $\begin{array}{c}1976 \\
\text { SI }\end{array}$ & $\begin{array}{c}2007 \\
\text { SIV }\end{array}$ & $\begin{array}{c}\text { Diferencia } \\
\text { SI-S IV }\end{array}$ & $\begin{array}{c}\text { Tasa anual \% } \\
\text { S I-S IV }\end{array}$ \\
\hline Bosques & 351036,0 & 342230,0 & -8806 & $-0,08$ \\
\hline Selvas & 349341,7 & 307757,7 & -41584 & $-0,41$ \\
\hline MA y ME & 633875,7 & 567082,6 & -66793 & $-0,36$ \\
\hline VH y OTV & 58930,3 & 54007,3 & -4923 & $-0,28$ \\
\hline ASV & 8102,5 & 9962,1 & 1860 & 0,67 \\
\hline Agropecuario & 527357,4 & 630393,1 & 103036 & 0,58 \\
\hline PF & 303,5 & 415,5 & 112 & 1,02 \\
\hline AH & 1993,9 & 16059,6 & 14066 & 6,96 \\
\hline CA & 20888,3 & 25275,7 & 4387 & 0,62 \\
\hline ACU & $5 / \mathrm{r}$ & 909,4 & 909 & \\
\hline S/INF & 2533,5 & 270,1 & -2263 & \\
\hline TOTAL & 1954363 & 1954363 & 0 & \\
\hline
\end{tabular}

MA matorral, ME mezquital, VH vegetación hidrófila, OTV otros tipos de vegetación, ASV áreas sin vegetación aparente, PF plantaciones forestales, AH asentamientos humanos, CA cuerpos de agua, ACU acuacultura, S/INF sin información. S I Serie I de INEGI, S IV Serie IV de INEGI. 
tercera parte (31,5\%) de la superficie ocupada en 1976 durante el periodo analizado.

A partir de los cambios registrados en la matriz de transición se calcularon las tasas de cambio para cada tipo de vegetación en el periodo S I - S IV (Tabla 4). Resalta la tasa de pérdida en selvas y matorrales y mezquitales, así como la tasa de incremento de asentamientos humanos, plantaciones forestales y áreas sin vegetación aparente.

Incorporamos los datos registrados por el Inventario Nacional Forestal 2000 (Palacio et al., 2000 y Mas et al., 2004) con la finalidad de hacer un corte intermedio en el periodo analizado y poder calcular las tasas de cambio durante los subperiodos, y de esa manera identificar si hay una diferencia en la velocidad de los cambios registrados en los subperiodos y en el periodo completo. En la tabla 5 se presentan las tasas de cambio calculadas para los subperiodos 1976-2000 y 2000-2007. En esa tabla se identifica que, efectivamente, hay un incremento en la velocidad de la pérdida de vegetación hidrófila y otros tipos de vegetación, así como de la superficie agropecuaria de 2000 a 2007, mientras que los bosques, selvas y matorrales y mezquitales presentan una desaceleración en la velocidad de la pérdida durante el mismo periodo, inclusive muestran tasas positivas. Resalta también el aumento de la velocidad de incremento de la superficie ocupada por plantaciones forestales y cuerpos de agua durante el mismo subperiodo.

En lo referente al análisis de la transición de la superficie con vegetación secundaria, se obtuvieron los siguientes resultados (Tabla 6): sesenta y cuatro por ciento de la superficie con vegetación secundaria en 1976 se mantuvo en esa misma condición en 2007. Veintiséis por ciento de la superficie con vegetación secundaria en 1976 fue deforestada para 2007 y solamente $10 \%$ de la superficie con vegetación secundaria en 1976 presentó una recuperación para 2007.

Por otro lado, analizando de dónde provenía la superficie identificada con vegetación secundaria en 2007, se

TABLA 5. Tasa anual de cambio por formación vegetal en los subperiodos 1976-2000 y 2000-2007. Las superficies se presentan en km².

\begin{tabular}{|c|c|c|c|c|c|c|c|}
\hline & \multicolumn{3}{|c|}{ INF } & \multirow[t]{2}{*}{ Diferencia } & \multirow[t]{2}{*}{ Diferencia } & \multirow{2}{*}{ Tasa anual \% } & \multirow{2}{*}{ Tasa anual \% } \\
\hline & 1976 & & 2007 & & & & \\
\hline & SI & 2000 & SIV & S I-2000 & 2000-S IV & S I-2000 & 2000-S IV \\
\hline Bosques & 351036 & 328271 & 342230 & -22765 & 13959 & $-0,28$ & 0,60 \\
\hline Selvas & 349341 & 307648 & 307758 & -41694 & 110 & $-0,53$ & 0,01 \\
\hline MA y ME & 633875 & 557872 & 567083 & -76004 & 9211 & $-0,53$ & 0,23 \\
\hline VH у OTV & 58930 & 74919 & 54007 & 15989 & -20912 & 1,01 & $-4,57$ \\
\hline ASV & 8102 & 10195 & 9962 & 2092 & -233 & 0,96 & $-0,33$ \\
\hline Agropecuario & 527357 & 645612 & 630393 & 118255 & -15219 & 0,85 & $-0,34$ \\
\hline PF & 303 & 226 & 416 & -78 & 190 & $-1,23$ & 9,12 \\
\hline $\mathrm{AH}$ & 1994 & 12430 & 16060 & 10436 & 3630 & 7,92 & 3,73 \\
\hline$C A$ & 20888 & 10991 & 25276 & -9897 & 14285 & $-2,64$ & 12,63 \\
\hline ACU & $s / r$ & $s / r$ & 909 & $s / r$ & 909 & $-0,28$ & 0,60 \\
\hline S/INF & 2533 & $s / r$ & 270 & $s / r$ & 270 & $-0,53$ & 0,01 \\
\hline TOTAL & 1954363 & 1948163 & 1954363 & & & & \\
\hline
\end{tabular}

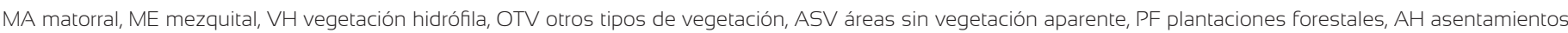
humanos, CA cuerpos de agua, ACU acuacultura, S/INF sin información. S I Serie I de INEGI, S IV Serie 4 de INEGI, INF inventario nacional forestal. 
TABLA 6. Transición de las superficies identificadas con vegetación secundaria en Serie I (1976) y Serie IV (2007) del INEGI.

\begin{tabular}{|c|c|c|c|c|c|}
\hline & $\begin{array}{c}\text { Superficie con } \\
\text { vegetación } \\
\text { secundaria en } \\
1976\left(\mathrm{~km}^{2}\right) \\
\end{array}$ & $\begin{array}{c}\text { \% de la superficie total } \\
\text { con vegetación } \\
\text { secundaria }\end{array}$ & & $\begin{array}{c}\text { Superficie con } \\
\text { vegetación } \\
\text { secundaria en } \\
2007\left(\mathrm{~km}^{2}\right) \\
\end{array}$ & $\begin{array}{c}\text { \% de la superficie } \\
\text { total con vegetación } \\
\text { secundaria }\end{array}$ \\
\hline $\begin{array}{c}\text { Permanencia en } \\
2007\end{array}$ & 205848 & 63,6 & $\begin{array}{c}\text { De vegetación } \\
\text { secundaria }\end{array}$ & 212669,7 & 50,0 \\
\hline $\begin{array}{l}\text { Deforestada en } \\
2007\end{array}$ & 83529 & 25,8 & $\begin{array}{c}\text { De vegetación } \\
\text { primaria alterada }\end{array}$ & 150734,5 & 35,4 \\
\hline $\begin{array}{c}\text { Recuperada en } \\
2007\end{array}$ & 34250 & 10,6 & $\begin{array}{c}\text { De vegetación } \\
\text { deforestada }\end{array}$ & 6211,0 & 1,5 \\
\hline & & & Recuperación & 55981,2 & 13,2 \\
\hline Total & 323626,8 & 100 & Total & 425596,4 & 100 \\
\hline
\end{tabular}

encontró que $50 \%$ de la misma permaneció en esa condición de la vegetación desde 1976, que 35\% de la superficie representó una alteración a la vegetación primaria de 1976, que $1,5 \%$ provino de procesos de deforestación y que solamente $13 \%$ respondió a un proceso de recuperación de la vegetación.

\section{DISCUSIÓN}

Los resultados del análisis para todo el periodo analizado (1976-2007) muestran que existen marcadas diferencias en la velocidad de cambio en cada tipo de vegetación, resaltando las tasas de incremento para plantaciones forestales y de decremento para selvas, así como la relativa estabilidad para los bosques. Sin embargo, es notorio que continúa la tendencia del cambio de uso de suelo forestal, en sus diferentes tipos, a usos agropecuarios, principalmente para la agricultura de temporal.

Los resultados presentados muestran que efectivamente las tasas de cambio calculadas para el periodo 1976-2007 son menores que las calculadas para los periodos 1976-2000 (Mas et al., 2004) y 1976-2002 (Semarnat, 2006), lo que indica que los ritmos de pérdida de vegetación forestal son más lentos.
Al hacer el análisis por subperiodos se identifica que las tasas de cambio para bosques, selvas y el agrupado de matorrales y mezquitales han pasado de ser negativas en el subperiodo 1976-2000 a positivas de 2000 a 2007. En el caso opuesto, se tiene que las tasas de cambio para las áreas sin vegetación aparente y usos agropecuarios, pasaron de ser positivas en el subperiodo 1976-2000 a negativas en 2000-2007. En el caso de los usos agropecuarios se puede especular sobre el efecto en los últimos seis años del periodo analizado de la ausencia de políticas gubernamentales que favorezcan la producción agropecuaria de los pequeños y medianos productores, además de los efectos de la migración rural, como lo señalan López et al. (2006), García-Barrios et al. (2009) y Toledo (2010). El caso de la vegetación hidrófila y otros tipos de vegetación es preocupante, tomando en cuenta que en ese grupo entran los manglares, ya que de una tasa positiva de $1 \%$ en el subperiodo 1976-2000, pasó a una tasa de pérdida anual de 4,6\% en el subperiodo 2000-2007.

Por otro lado, resalta el incremento de la tasa de plantaciones forestales (en particular entre el 2002 y el 2007, según el reporte de FAO, 2010) y cuerpos de agua en el periodo 2000-2007. Este incremento contrasta con el caso de los usos agropecuarios, que se contrajo a una tasa de 
0,34\% anual, ya que durante ese subperiodo existieron marcadas políticas federales para la construcción de presas y el impulso a la reforestación, principalmente con un enfoque de restauración.

Otro resultado interesante fue la disminución en la velocidad del incremento de la superficie ocupada por asentamientos humanos, que para el subperiodo 20002007 (3,73\%) disminuyó a la mitad de la tasa de incremento publicada para el subperiodo 1976-2000 (7,92\%).

Comparando el análisis por subperiodos realizado en este trabajo con los realizados en estudios previos (Velázquez et al., 2002 y Semarnat, 2006), se identificó la tendencia en la disminución de la pérdida de vegetación forestal, inclusive con una tasa anual positiva para bosques y prácticamente en equilibrio para las selvas durante el subperiodo 2000-2007.

En cuanto al análisis de la evolución de los polígonos etiquetados con vegetación secundaria en la Serie I (1976) y la Serie IV (2007) del INEgI, no cabe duda que se ha incrementado la superficie ocupada por esa condición de la vegetación en más de 30\%, y que se continúan alterando más de 500000 ha al año en promedio. El análisis fino realizado en este trabajo ha identificado que solamente $13 \%$ de esa condición de vegetación existente en 2007 se debe a una recuperación, por lo que es completamente incorrecta la práctica de descontar a la superficie total deforestada la superficie ocupada por vegetación secundaria identificada desde la publicación de Semarnat (2006), y que se ha mantenido hasta el documento de Conafor (2012b), lo que conlleva a una subvaluación de las tasas de deforestación y la superficie total deforestada en los documentos oficiales.

Los resultados obtenidos muestran que $64 \%$ de la superficie con vegetación secundaria en 1976 se mantuvo con esa condición en 2007, lo que indica que esas áreas están sujetas a alteraciones constantes que evitan que se recupere la vegetación forestal, pero no lo suficientemente intensas para que pasen a ser áreas deforestadas. Esa situación de deforestación sobre superficies con vegetación secundaria en 1976 se dio en 26\% de ellas en 2007. Además, para fortalecer el dato de que solamente $13 \%$ de la vegetación secundaria identificada en 2007 proviene de procesos de recuperación, $10 \%$ de la superficie con vegetación secundaria en 1976 se recuperó para 2007.

Con esos resultados se puede pensar que los datos correctos de la tabla 7.7 presentados por Semarnat (2006) deben ser 898403 ha anuales deforestadas durante el periodo 1976-1993 y 505004 ha anuales deforestadas en el periodo 1993-2002. En el caso de los datos presentados por Conafor (2012b), la cifra correcta debe ser 511789 ha al año para el periodo 2000-2005, tomando en cuenta que sólo $13 \%$ de la superficie ocupada por bosques y selvas con vegetación secundaria se recuperó, en lugar de las 235000 ha al año publicadas para bosques y selvas en ese periodo.

Ahora, para la cifra indicada por la Presidencia de la República en 2011 y 2012, así como en el informe del país (FAO, 2010), no hay datos derivados de estudios nacionales (el más reciente es la Serie IV de INEGI), sino que es una proyección generada a partir de las tendencias identificadas en la última década (Conafor, 2012a), pero acarreando el "error" de descontar 100\% de la vegetación secundaria existente que ha sido una práctica común desde 2006.

Si se toma en cuenta el promedio anual de incremento de la superficie ocupada por la vegetación secundaria en bosques y selvas (318 $148 \mathrm{ha}$ ) en la Serie IV de INEGI, y se asume $13 \%$ de recuperación encontrado en este trabajo, tenemos que la cifra "real" que debió informar la Presidencia de la República en 2011 y 2012, así como la FAO en su informe de México 2010 es 431789 ha de bosques y selvas deforestadas al año.

Lo que es evidente es que la deforestación no ha disminuido, en todo caso se ha estabilizado alrededor de las 500000 ha al año, situación que ya había sido detectada por Mas et al. (2009), además de que la superficie ocupada por vegetación secundaria y plantaciones forestales se ha incrementado desde el año 2000.

\section{CONCLUSIONES}

La deforestación en México no ha disminuido, en contraste con lo que indican las cifras oficiales más recientes. 
En todo caso, se ha estabilizado alrededor de 500000 ha anuales entre 1993 y 2007.

En este trabajo se encontró para el periodo 19762007 una superficie anual deforestada de 534707 ha, con una tasa anual de $-0,08 \%$ para bosques, $-0,41 \%$ para selvas y $-0,36 \%$ para matorrales y mezquitales juntos.

La velocidad en la pérdida de la vegetación forestal en bosques y selvas ha disminuido desde el año 2000, en comparación con las tasas de cambio calculadas en periodos anteriores. Se encontró una tasa de incremento anual de $0,6 \%$ para bosques y una situación de estabilidad en las selvas, con una tasa de incremento anual de $0,01 \%$.

El incremento de la superficie ocupada por vegetación secundaria es mayor en el periodo 2000-2007 que en periodos previos.

La evolución de los polígonos con vegetación secundaria muestra que, en promedio, $10 \%$ de la superficie con esa condición se regenera en bosques primarios.

En 2007, 13\% de la superficie con vegetación secundaria es producto de procesos de recuperación de la vegetación, mientras que $50 \%$ se mantienen en esa condición desde 1976 y $35 \%$ es producto de la alteración de vegetación primaria.

Los datos oficiales publicados, de pérdida de vegetación forestal, están subvaluados, ya que descuentan $100 \%$ de la superficie ocupada por la vegetación secundaria, práctica que fue claramente detectada a partir del año 2006.

Es necesario que en los próximos informes oficiales se elimine la práctica de descontar el $100 \%$ de la superficie ocupada por la vegetación secundaria a la superficie deforestada, y que se realice un análisis de la dinámica de los polígonos identificados con vegetación secundaria para poder informar una cifra de deforestación más cercana a la realidad.

\section{REFERENCIAS}

Álvarez-Icaza, P., G. Cervera, C. Garibay, P. Gutiérrez y F. Rosete. 1993. Los umbrales del deterioro. La dimensión ambiental de un desarrollo desigual en la región purépe- cha. Facultad de Ciencias. UNAM. PAIR-UNAM, Friederich Ebert Stiftung. México. 274 p.

Bocco, G., M. Mendoza y O. Masera. 2001. La dinámica del cambio del uso del suelo en Michoacán. Investigaciones Geográficas 44:18-38.

Brooks, T.M., R.A. Mittermeier, G.A.B. da Fonseca, J. Gerlach, M. Hoffmann, J.F. Lamoreux, C.G. Mittermeier, J.D. Pilgrim y A.S.L. Rodrígues. 2006. Global Biodiversity Conservation priorities. Science 313:58-61.

CABAL, S.A. 2010. Bosques, deforestación y monitoreo de carbono: una valoración potencial de REDD+ en Mesoamérica. 89 p.

Campos, C.P., M.S. Muylaert y L. Pinguelli. 2004. Historical $\mathrm{CO}_{2}$ emission and concentrations due to land use change of croplands and pastures by country. Science of the Total Environment 346:149-155.

Castillo, P.E., P. Lehtonen, M. Simula, V. Rosa y R. Escobar. 1989. Proyecciones de los principales indicadores forestales de México a largo plazo (1988-2012). Reporte interno. Subsecretaría forestal. Cooperación México-Finlandia. SARH. México.

Chikhaoui, M., F. Bonn, A.I. Bokoye y A. Merzouk. 2005. A spectral index for land degradation mapping using ASTER data: Application to a semi-arid Mediterranean catchment. International Journal of Applied Earth Observation and Geoinformation 7:140-153.

Clark, J.S. 2009. Beyond neutral science. Trends in ecology and evolution 24(1):8-15.

Conafor (Comisión Nacional Forestal). 2012a. Inventario Nacional Forestal y de Suelos. Informe 2004-2009. Zapopan, México. 173 p.

Conafor (Comisión Nacional Forestal). 2012b. Propuesta de actualización del Programa Estratégico Forestal para México 2025. Borrador interno. Zapopan, México. 142 p.

Cortez-Ortiz, A. 1990. Estudio preliminar sobre deforestación en la región fronteriza del río Usumacinta. Reporte interno. INEGI. México, D. F.

Cortina, S., P. M. Mendoza e Y. Ogneva-Himmelberger. 1999. Cambios en el uso de suelo y deforestación en el sur de los estados de Campeche y Quintana Roo, México. Investigaciones Geográficas 38:41-56. 
Cuarón O., A.D. 1991. Conservación de los primates y de sus habitats en el sur de México. Tesis de Maestría. Universidad Nacional de Heredia. Costa Rica.

Cuevas, M.L., A. Garrido, J.L. Pérez y D.I. González. 2010. Procesos de cambio de uso de suelo y degradación de la vegetación natural. In: Cotler, H., coord. Las cuencas hidrográficas de México. Diagnóstico y priorización. Semarnat, INE y Fundación G. Río Arronte I.A.P. México, D.F. p:96-103.

Dai, A. 2011. Drought under global warming: a review. Wiley Interdisciplinary Reviews: Climate Change 2(1):45-65.

Dirzo, R. y M.C. García. 1991. Rates of deforestation in Los Tuxtlas a Neotropical area in southeast Mexico. Conservation Biology 6:84-90.

Dunjó G., G. Pardini y M. Gispert. 2003. Land use change effects on abandoned terraced soils in a Mediterranean catchment, NE Spain. Catena 52:23-37.

Ehrlich, P. y A. Ehrlich. 2013. Can a collapse of global civilization be avoided? Proceedings of the Royal Society B. 280(1754):2012-2845.

FAO (Organización de las Naciones Unidas para la Alimentación y la Agricultura). 1988. An interim report of on the state of forest resources in the developing countries. Forest Resource Division. Forestry Department. Roma.

FAO (Organización de las Naciones Unidas para la Alimentación y la Agricultura). 1995. Forest resources 1990. FAO. Roma.

FAO (Organización de las Naciones Unidas para la Alimentación y la Agricultura). 1996. Forest resources assessment 1990. Survey of tropical forest cover and study of change processes. FAO. Roma.

FAO (Organización de las Naciones Unidas para la Alimentación y la Agricultura). 1997. State of the world forest 1997. FAO, Roma.

FAO (Organización de las Naciones Unidas para la Alimentación y la Agricultura). 2005. Evaluación de los recursos forestales mundiales 2005. México. Informe nacional núm. 189. Roma. 68 p.

FAO (Organización de las Naciones Unidas para la Alimentación y la Agricultura). 2010. Evaluación de los recursos forestales mundiales 2010. Informe Nacional México. FRA2010/132. Roma. 98 p.
García-Barrios, L., Y.M. Galván-Miyoshi, I.A. ValdiviesoPérez, O.R. Masera, G. Bocco y J. Vandermeer. 2009. Neotropical forest conservation, agricultural intensification, and rural out-migration: The mexican experience. Bioscience 59(10):863-873.

Garibay, C. y G. Bocco. 2012. Cambios de uso de suelo en la meseta purépecha (1976-2005). INE-Semarnat y CIGAUNAM. México, D.F. 124 p.

Heistermann, M., C. Müller, y K. Ronneberger. 2006. Land in sight? Achievements, deficits and potentials of continental to global scale land-use modeling. Agriculture, Ecosystems and Environment 114:141-158.

INEGI. 2000. Diccionario de datos de uso de suelo y vegetación: Escala 1:250 000 (vectorial). Serie I. DGG-INEGI. México. INEGI. 2005. Conjunto Nacional de Uso del Suelo y Vegetación a escala 1:250 000. Serie III. DGG-INEGI. México.

INEGI. 2010. Conjunto Nacional de Uso del Suelo y Vegetación a escala 1:250 000. Serie IV. DGG-INEGI. México.

Jianchu, X., J. Fox, J.B. Vogler, Z. Peifang, F. Yongshou, Y. Lixin, Q. Jie, y S. Leisz. 2005. Land-Use and Land-Cover Change and Farmer Vulnerability in Xishuangbanna Prefecture in Southwestern China. Environmental Management 36(3):404-413.

Kerr, S., S. Liu, A.S.P. Pfaff y R.F. Hughes. 2003. Carbon dynamics and land-use choices: building a regional-scale multidisciplinary model. Journal of Environmental Management 69:25-37.

Lambin, E.F. y H. Geist, eds. 2006. Land-use and land-cover change: local processes and global impacts. Springer-Verlag. Berlin. 222 p.

López, E., G. Bocco, M. Mendoza, A. Velázquez y J.R. Aguirre. 2006. Peasant emigration and land-use change at the watershed lever: A GIS-based approach in Central Mexico. Agricultural Systems 90:62-78.

López, E., G. Bocco, M. Mendoza y E. Duhau. 2001. Predicting land-cover and land-use change in the urban fringe. A case in Morelia city, Mexico. Landscape and Urban Planning 55:271-285.

Mas, J.F., A. Velázquez y S. Couturier. 2009. La evaluación de los cambios de cobertura/uso del suelo en la República Mexicana. Investigación Ambiental 1(1):23-39. 
Mas, J.F., A. Velásquez, J. Reyes, R. Mayorga-Saucedo, C. Alcántara, G. Bocco, R. Castro, T. Fernández y A. PérezVega. 2004. Assessing land use/cover changes: a nationwide multidate spatial database for Mexico. International Journal of Applied Earth Observation and Geoinformation 5(4):249-338.

Mas, J.F., A. Velásquez, J.L. Palacio, G. Bocco, A. Peralta y J. Prado. 2002. Assessing forest resources in Mexico: wallto-wall land use/cover mapping. Photogrammetric Engineering and Remote Sensing 68(10):966-968.

Mas, J.F., V. Sorani y R. Álvarez. 1996. Elaboración de un modelo de simulación del proceso de deforestación. Investigaciones Geográficas núm. esp. 5:43-57.

Masera, O., M.J. Ordóñez y R. Dirzo. 1992. Emisiones de carbono a partir de la deforestación en México. Ciencia 43:151-153

Masera, O. 1996. Deforestación y degradación forestal en México. Documentos de trabajo núm. 19. GIRA A. C. Pátzcuaro, México. 52 p.

Milesi, C., H. Hashimoto, S.W. Running y R.R. Nemani. 2005. Climate variability, vegetation productivity and people at risk. Global and Planetary Change 47:221-231.

Musaoglu, N., A. Tanik y V. Kocabas. 2005. Identification of land-cover changes through image processing and associated impacts on water reservoir conditions. Environmental Management 35(2):220-230.

Myers, N. 1989. Deforestation rates in tropical forests and their climate implications. Friends of the Hearth, Gran Bretaña. 116 p.

Palacio-Prieto, J.L.; G. Bocco; A. Velázquez, J.F. Mas; F. Takaki-Takaki; A. Victoria; L. Luna-González; G. Gómez-Rodríguez; J. López-García; M. Palma; I. TrejoVázquez; A. Peralta; J. Prado-Molina; A. Rodríguez; R. Mayorga-Saucedo y F. González. 2000. La condición actual de los recursos forestales en México: resultados del Inventario Nacional Forestal 2000. Investigaciones Geográficas 43:183-203.

Presidencia de la República. 2012. Quinto Informe de Ejecución del Plan Nacional de Desarrollo 2007-2012. Presidencia de la República. México, D. F. 828 p.
Presidencia de la República. 2011. Cuarto Informe de Ejecución del Plan Nacional de Desarrollo 2007-2012. Presidencia de la República. México, D.F. 787 p.

Repetto, R. 1988. The forests for the trees? Governement policies and the misuse of forest resources. World Resources Institute. Washington D.C., EUA.

Rosete, F. 2008. Modelos predictivos de cambio de uso del suelo en la Península de Baja. California, México. Tesis de doctorado. Facultad de Filosofía y Letras. UNAM. México. $152 \mathrm{p}$.

Rosete, F., J.L. Pérez-Damián y G. Bocco. 2008. Cambio de uso el suelo y vegetación en la Península de Baja California, México. Investigaciones Geográficas 67:39-58.

Rosete, F., J.A. Ordóñez y O. Masera. 1997. Dinámica del cambio de uso del suelo y emisiones de carbono en la meseta purépecha. Reporte interno. Instituto de Ecología. UNAM. México. 25 p.

Rudel, K. T., Coomes, O. T., Moran, E., Hard, F., Angelsen, A., Xu, J., Lambin, E. 2005. Forest transitions: towards a global understanding of land use change. Global Environmental Change 15: 23-31.

Salitchev, K. 1981. Cartografía. Editorial Pueblo y Educación. La Habana, Cuba.

Sánchez, J., G. Bocco, J. Fuentes y A. Velázquez. 2003. Análisis de la cobertura y uso del terreno en el contexto de su dinámica espacio-temporal. In: Velázquez, A., A. Torres y G. Bocco, comp. Las enseñanzas de San Juan. Investigación participativa para el manejo integral de recursos naturales. INE-Semarnat. México, D.F. p:235-256.

Sánchez, S., A. Flores, I.A. Cruz-Leyva y A. Velázquez. 2009. Estado y transformación de los ecosistemas terrestres por causas humanas. In: Dirzo, R., González R. e I.J. March, comp. El capital natural de México: Estado de conservación y tendencias de cambio. Volumen II. Conabio, México.

SARH (Secretaría de Agricultura y Recursos Hidráulicos). 1984. Comisión del Plan Nacional Hidráulico 1984: Desarrollo Rural Integral de la Selva Lacandona, México. SARH. México, D.F.

Semarnat (Secretaría de Medio Ambiente y Recursos Naturales). 2006. La Gestión Ambiental en México. Semarnat. México, D. F. México. 468 p. 
Semarnat (Secretaría de Medio Ambiente y Recursos Naturales). 2007. Programa sectorial de medio ambiente y recursos naturales 2007-2012. México, D.F. 172 p.

Spurr, H. y B. Barnes. 1982. Ecología forestal. AGT editores. Primera edición en español. México, D.F. 690 p.

Srivastava, S. D. 2002. The role of conservation in expanding biodiversity research. Oikos 98 (2):351-360.

Toledo, A. 2010. Globalización, Migración y Ambiente. INAP. México, D.F. México. 128 p.

Toledo, V.M. 1989. Bio-economic costs of transforming tropical forest to pastures in Latinoamerica. In: Hecht, S.,ed. Cattle ranching and tropical deforestation in Latinoamerica. Westview Press. Boulder, Colorado.

Trejo I. y R. Dirzo. 2000. Deforestation of seasonally dry tropical forest a national and local analysis in Mexico. Biological Conservation 94(2):133-142.

Velázquez, A. 2008. La dinámica de la cubierta forestal de México. Página de la Sociedad Mexicana de Física (http:// www.smf.mx/C-Global/webCubFor.htm).

Velázquez, A., J.F. Mas, G. Bocco y J.L. Palacio-Prieto. 2010. Mapping land cover changes in Mexico, 1976-2000 and applications for guiding environmental management policy. Singapore Journal of Tropical Geography 31:152-162.
Velázquez, A., E. Durán, I. Ramírez, J.F. Mas, G. Bocco, G. Ramírez y J.L. Palacio. 2003. Land-use cover change processes in highly biodiverse areas: the case of Oaxaca, Mexico. Global Environmental Change 13:175-184.

Velázquez, A., J.F. Mas, J.R. Díaz, R. Mayorga-Saucedo, P.C. Alcántara, R. Castro, T. Fernández, G. Bocco, E. Escurra y J.L. Palacio. 2002. Patrones y tasas de cambio de uso del suelo en México. Gaceta Ecológica 62:21-37.

WRI (World Resources Institute). 1992. World Resources 199293. Oxford University Press. Nueva York.

WRI (World Resources Institute). 1994. World Resources 199495. Oxford University Press. Nueva York.

Xiao J., Y. Shen, J. Ge, R. Tateishi, C. Tang, Y. Liang y Z. Huang. 2006. Evaluating urban expansion and land use change in Shijiazhuang, China, by using GIS and remote sensing. Landscape and Urban Planning 75:69-80.

Manuscrito recibido el 7 de mayo de 2013.

Aceptado el 26 de septiembre de 2013.

Este documento se debe citar como:

Rosete-Vergés, F.A., J.L. Pérez-Damián, M. Villalobos-Delgado, E.N. Navarro-Salas, E. Salinas-Chávez y R. Remond-Noa. 2014. El avance de la deforestación en México 1976-2007. Madera y Bosques 20:21-35. 